\title{
STOCHASTIC BROWNIAN GAME OF ABSOLUTE DOMINANCE
}

\author{
SHANGZHEN LUO, ${ }^{*}$ University of Northern Iowa
}

\begin{abstract}
In this paper we study a reinsurance game between two insurers whose surplus processes are modeled by arithmetic Brownian motions. We assume a minimax criterion in the game. One insurer tries to maximize the probability of absolute dominance while the other tries to minimize it through reinsurance control. Here absolute dominance is defined as the event that liminf of the difference of the surplus levels tends to $-\infty$. Under suitable parameter conditions, the game is solved with the value function and the Nash equilibrium strategy given in explicit form.
\end{abstract}

Keywords: Stochastic differential game; Nash equilibrium; diffusion approximation; reinsurance; absolute dominance

2010 Mathematics Subject Classification: Primary 60G40

Secondary 93E20

\section{Introduction}

Game theory is used to model and analyze interactive situations in various environments. It has been widely applied in economics and insurance. In [3], a finite-horizon stochastic differential game is considered. It was proved that the sup-value and sub-value functions of the game are the unique viscosity solutions of the Bellman-Isaacs partial differential equations. In [1], portfolio games between two investors were considered. Conditions under which a game, with a general payoff function, has an achievable value were provided, and explicit representations of the value function and the equilibrium portfolio strategy were given. In [5], a two-player stochastic differential game was studied in a Lévy market, where Hamilton-JacobiBellman-Isaacs (HJBI) conditions were proved and the results applied to risk minimization problems. In [9], a proportional reinsurance game was formulated by maximizing or minimizing the exit probability of an interval. The value function and Nash equilibrium strategy were obtained explicitly. In [8], a similar game was studied with consideration of nonproportional reinsurance.

In this paper we study a game between two insurers with reinsurance control. We apply the modeling features of [1], [4], and [9], and formulate a competition between the two insurers using a two-player noncooperative differential game. The surplus processes are modeled by arithmetic Brownian motions with risk-free investment and Brownian perturbation. In this game, the insurers can purchase proportional reinsurance to control their risk exposure levels. That is, the drift and diffusion terms of the arithmetic Brownian motions can be reduced proportionally at the same time. We consider absolute dominance as the win scenario of the game. Here absolute dominance is defined as the event that one insurer eventually outperforms the other in surplus level, i.e. the liminf of the difference of the surplus levels tends to $-\infty$.

Received 6 February 2013; revision received 5 August 2013.

* Postal address: Department of Mathematics, University of Northern Iowa, Cedar Falls, IA 50614-0506, USA.

Email address: luos@uni.edu 
This scenario is motivated by the idea of absolute ruin (see [8]) or infinite time ruin (see [6]). To define the value function of the game, we use a minimax criterion: one insurer tries to maximize the probability of its absolute dominance while the other tries to minimize the probability (of being absolutely dominated) using reinsurance control. The value function is defined as the probability of absolute dominance under the optimal reinsurance control or play (if it exists for both players). If the value function is well defined and has proper smoothness conditions, it can be characterized by Fleming-Bellman-Isaacs (FBI) equations that involve supremum and infimum operations of a differential operator. Solvability of the game, in the case where the surplus processes are positively correlated, is studied in detail. We find that, under suitable parameter conditions, the FBI equations can be solved explicitly. By a verification result, the solution is shown to coincide with the value function. The saddle-point control strategy (or the Nash equilibrium strategy) is also found explicitly in these cases.

The rest of the paper is organized as follows. In Section 2 we formulate the problem. In Section 3 we state the FBI equations and prove the verification theorem. In Section 4 we solve the FBI equations. We give concluding remarks in Section 5.

\section{A noncooperative game and the value function}

In this section we formulate the game and define the value function. We begin with the diffusion approximation model under which the surplus processes are approximated by the following drifted Brownian motions (see, e.g. [2] and [7]):

$$
\mathrm{d} S_{1}=\mu_{1}(a) \mathrm{d} t+\sigma_{1}(a) \mathrm{d} w_{1}, \quad \mathrm{~d} S_{2}=\mu_{2}(b) \mathrm{d} t+\sigma_{2}(b) \mathrm{d} w_{2},
$$

where $\left\{w_{i}\right\}_{t \geq 0}, i=1,2$, are standard Brownian motions adapted to the information filtration $\left\{\mathcal{F}_{t}\right\}_{t \geq 0}$ in a probability space $(\Omega, \mathcal{F}, \mathbb{P})$ with $\mathbb{E}\left(\mathrm{d} w_{1} \mathrm{~d} w_{2}\right)=\rho \mathrm{d} t$ for some correlation coefficient $\rho$, and the drift and diffusion terms are given by

$$
\begin{array}{ll}
\mu_{1}(a)=\mu_{1} a-\lambda_{1}(1-a)=\left(\mu_{1}+\lambda_{1}\right) a-\lambda_{1}, & \sigma_{1}(a)=\sigma_{1} a, \\
\mu_{2}(b)=\mu_{2} b-\lambda_{2}(1-b)=\left(\mu_{2}+\lambda_{2}\right) b-\lambda_{2}, & \sigma_{2}(b)=\sigma_{2} b,
\end{array}
$$

which are based on proportional reinsurance (see [4] and [7]), where, for $i=1,2, \mu_{i}(>0)$, are insurance premium rates, $\lambda_{i}(\geq 0)$ are levels of reinsurance safety loading, $\sigma_{i}(>0)$ are volatilities of the Brownian motions which represent the risk levels, and $0 \leq a \leq 1$ and $0 \leq b \leq 1$ are levels of risk exposure. Write $\gamma_{i}=\mu_{i}+\lambda_{i}, i=1,2$, which represent the reinsurance premium rates.

Now we include risk-free investment and Brownian perturbation in the model. That is, the insurers earn or pay interest at a constant rate when the surplus levels are positive or negative, respectively, and the surplus levels are perturbed by Brownian noise. Hence, the surplus processes are governed by the Itô dynamics

$$
\begin{aligned}
& \mathrm{d} S_{1}=\left[r S_{1}+\mu_{1}(a)\right] \mathrm{d} t+\sigma_{1}(a) \mathrm{d} w_{1}+\mathrm{d} Z_{1}, \\
& \mathrm{~d} S_{2}=\left[r S_{2}+\mu_{2}(b)\right] \mathrm{d} t+\sigma_{2}(b) \mathrm{d} w_{2}+\mathrm{d} Z_{2},
\end{aligned}
$$

where $Z_{i}, i=1,2$, are zero-mean Brownian motions that model the perturbing terms, and $r$ is the constant interest rate.

Next we consider stochastic reinsurance control, with which the risk exposure levels can be changed dynamically over time. We denote by $S_{1}^{A}$ the controlled surplus process of the first insurer under the dynamic reinsurance control with risk exposure process $A:=\left\{a_{t}\right\}_{t \geq 0}$, and 
by $S_{2}^{B}$ the controlled surplus process of the second insurer under the control with risk exposure process $B:=\left\{b_{t}\right\}_{t \geq 0}$. The difference in the surplus processes, $X^{A, B}:=S_{1}^{A}-S_{2}^{B}$, is governed by the stochastic differential equation

$$
\mathrm{d} X_{t}^{A, B}=\left[r X_{t}^{A, B}+\gamma_{1} a_{t}-\gamma_{2} b_{t}-\left(\lambda_{1}-\lambda_{2}\right)\right] \mathrm{d} t+\sigma_{1} a_{t} \mathrm{~d} w_{1}-\sigma_{2} b_{t} \mathrm{~d} w_{2}+\sigma \mathrm{d} Z
$$

with $X_{0}=x$, where $x$ is the initial difference, $Z$ is a standard Brownian motion independent of $w_{1}$ and $w_{2}$, and $\sigma(>0)$ is the volatility of the perturbing Brownian motion.

A control policy with risk exposure process $A$ is said to be admissible if

(i) $0 \leq a_{t} \leq 1$;

(ii) $a_{t} \in \mathcal{F}_{t}$ for all $t>0$; and

(iii) $a_{t}$ is square integrable over $[0, T]$ for all $T>0$ almost surely $\left(\int_{0}^{T} a_{t}^{2} \mathrm{~d} t\right.$ exists).

We denote by $\pi$ the set of admissible controls.

Now we define the performance function of the game under a paired admissible policy $(A, B)$ as

$$
V^{A, B}(x):=\mathbb{P}_{x}\left(\liminf _{t \rightarrow \infty} X_{t}^{A, B}=-\infty\right),
$$

where $\mathbb{P}_{x}(\cdot)=\mathbb{P}\left(\cdot \mid X_{0}=x\right)$. The performance function is the probability that insurer two attains absolute dominance, or, in other words, that liminf of the difference in the surplus levels tends to $-\infty$. We note that policies $A$ and $B$ are adapted to the same information filtration $\mathcal{F}$. This implies that there is complete observation in the game. That is, insurer one's strategy is instantaneously observed by insurer two, and vice versa.

In the following we apply a minimax criterion to define the value function. We first need to define sub-value and sup-value functions of the game:

$$
\underline{V}(x):=\sup _{B \in \pi} \inf _{A \in \pi} V^{A, B}(x), \quad \bar{V}(x):=\inf _{A \in \pi} \sup _{B \in \pi} V^{A, B}(x) .
$$

The sub-value function is obtained in a way such that insurer one plays first to minimize the probability of absolute dominance of insurer two and insurer two plays second to maximize it. The sup-value function is obtained in the opposite way. Obviously, it holds that $\underline{V}(x) \leq \bar{V}(x)$ for all $x \in(-\infty, \infty)$. In the case $\underline{V}(x)=\bar{V}(x)$ for all $x \in(-\infty, \infty)$, we define the value function as

$$
V(x)=\underline{V}(x)=\bar{V}(x) .
$$

Note that $\underline{V}$ and $\bar{V}$ are decreasing functions and $V$ is a decreasing function when it exists.

\section{FBI equations and the verification theorem}

In this section we give the FBI equations and prove the verification theorem. Suppose that the value function of the game exists and is a $C^{2}(-\infty, \infty)$ function. Furthermore, suppose that there exists a so-called admissible Nash equilibrium or saddle-point strategy, denoted by $\left(A^{*}, B^{*}\right)$, that satisfies

$$
V^{A^{*}, B}(x) \leq V^{A^{*}, B^{*}}(x) \leq V^{A, B^{*}}(x)
$$

for any $x \in(-\infty, \infty)$ and admissible controls $A$ and $B$. With Markov decision making, the saddle-point strategy $\left(A^{*}, B^{*}\right)$ is a feedback strategy determined by a pair of risk exposure functions $\left(a^{*}(x), b^{*}(x)\right)$. That is, the reinsurance controls at any time are given by $a^{*}(x)$ 
and $b^{*}(x)$ which depend only on the then-current surplus difference $x$. By a rather standard procedure, we can show that the value function $V$ solves the FBI equations

$$
\sup _{b \in[0,1]} L^{a^{*}(x), b} V(x)=0, \quad \inf _{a \in[0,1]} L^{a, b^{*}(x)} V(x)=0,
$$

where the differential operator $L$ is defined by

$L^{a, b} V(x)=\left[r x+\gamma_{1} a-\gamma_{2} b-\left(\lambda_{1}-\lambda_{2}\right)\right] V^{\prime}(x)+\frac{1}{2}\left(\sigma_{1}^{2} a^{2}+\sigma_{2}^{2} b^{2}-2 \rho \sigma_{1} \sigma_{2} a b+\sigma^{2}\right) V^{\prime \prime}(x)$, and the functions $a^{*}(x)$ and $b^{*}(x)$ satisfy

$$
a^{*}(x)=\arg \inf _{a \in[0,1]} L^{a, b^{*}(x)} V(x), \quad b^{*}(x)=\arg \sup _{b \in[0,1]} L^{a^{*}(x), b} V(x) .
$$

Note that the pair $\left(a^{*}(x), b^{*}(x)\right)$ is a saddle point of $L^{a, b} V(x)$ and it holds that

$$
L^{a^{*}(x), b^{*}(x)} V(x)=0 .
$$

We skip the derivation of the FBI equations. In the following we prove the verification theorem, which shows that if a decreasing $C^{2}$ solution to (3.1) with boundary conditions

$$
V(\infty)=0 \quad \text { and } \quad V(-\infty)=1
$$

exists, then the value function exists and coincides with the solution.

First we give the result that the controlled process exits any finite interval with probability 1 . For any $M, N$ with $-\infty<M<x<N<\infty$ and any admissible controls $A$ and $B$, define the first hitting times

$$
\tau_{M}^{A, B}=\inf \left\{t>0: X^{A, B}(t)=M\right\}, \quad \tau_{N}^{A, B}=\inf \left\{t>0: X^{A, B}(t)=N\right\},
$$

and

$$
\tau_{M, N}^{A, B}=\tau_{M}^{A, B} \wedge \tau_{N}^{A, B} .
$$

Lemma 3.1. For any $-\infty<M<x<N<\infty$ and any admissible controls $A$ and $B$, it holds that $\mathbb{P}_{x}\left(\tau_{M, N}^{A, B}<\infty\right)=1$.

Proof. For convenience, write $\tau=\tau_{M, N}^{A, B}$ and

$$
\xi_{t}=r X_{t}^{A, B}+\gamma_{1} a_{t}-\gamma_{2} b_{t}-\left(\lambda_{1}-\lambda_{2}\right), \quad \eta_{t}^{2}=\sigma_{1}^{2} a_{t}^{2}-2 \rho \sigma_{1} \sigma_{2} a_{t} b_{t}+\sigma_{2}^{2} b_{t}^{2}+\sigma^{2} .
$$

Applying Itô's formula to $\mathrm{e}^{K X_{t}^{A, B}}$, we obtain

$$
\begin{aligned}
\mathrm{e}^{K X_{\tau \wedge T}^{A, B}-\mathrm{e}^{K x}=} & \int_{0}^{\tau \wedge T} K \mathrm{e}^{K X_{t}^{A, B}}\left(\frac{1}{2} K \eta_{t}^{2}+\xi_{t}\right) \mathrm{d} t+\sigma_{1} \int_{0}^{\tau \wedge T} K \mathrm{e}^{K X_{t}^{A, B}} a_{t} \mathrm{~d} w_{1} \\
& -\sigma_{2} \int_{0}^{\tau \wedge T} K \mathrm{e}^{K X_{t}^{A, B}} b_{t} \mathrm{~d} w_{2}+\sigma \int_{0}^{\tau \wedge T} K \mathrm{e}^{K X_{t}^{A, B}} \mathrm{~d} Z_{t}
\end{aligned}
$$

Taking the expectation of both sides, we have

$$
\mathbb{E}_{x}\left[\mathrm{e}^{\left.K X_{\tau \wedge T}^{A, B}\right]-\mathrm{e}^{K x}}=\mathbb{E}_{x}\left[\int_{0}^{\tau \wedge T} K \mathrm{e}^{K X_{t}^{A, B}}\left(\frac{1}{2} K \eta_{t}^{2}+\xi_{t}\right) \mathrm{d} t\right] .\right.
$$


Since $\xi_{t}$ is bounded for $t \in(0, \tau)$ and $2 \sigma_{1}^{2}+2 \sigma_{2}^{2}+\sigma^{2} \geq \eta_{t}^{2} \geq \sigma^{2}$, we can choose a negative $K$ with large $|K|$ such that $\frac{1}{2} K \eta_{t}^{2}+\xi_{t}<-1$ for all $t \in(0, \tau)$. From (3.4), it holds that

$$
\begin{aligned}
\mathrm{e}^{K M} & \geq \mathbb{E}_{x}\left[\mathrm{e}^{\left.K X_{\tau \wedge T}^{A, B}\right]}\right. \\
& \geq K \mathbb{E}_{x}\left[\int_{0}^{\tau \wedge T} \mathrm{e}^{K X_{t}^{A, B}}\left(\frac{1}{2} K \eta_{t}^{2}+\xi_{t}\right) \mathrm{d} t\right] \\
& \geq-K \mathbb{E}_{x}\left[\int_{0}^{\tau \wedge T} \mathrm{e}^{K X_{t}^{A, B}} \mathrm{~d} t\right] .
\end{aligned}
$$

Thus,

$$
\mathrm{e}^{K M} \geq-K \mathbb{E}_{x}\left(\mathbf{1}_{\{\tau>T\}} \int_{0}^{T} \mathrm{e}^{K X_{t}^{A, B}} \mathrm{~d} t\right) \geq-K \mathrm{e}^{K N} T \mathbb{P}_{x}(\tau>T) .
$$

Letting $T \rightarrow \infty$, we obtain $\mathbb{P}_{x}(\tau=\infty)=0$.

Below we show that certain events (e.g. the controlled process never hits $y$ given it starts at $x$ ) occur with positive probability.

Lemma 3.2. For any $-\infty<M<x<N<\infty$ and any admissible controls $A$ and $B$, it holds that

(i) $0<\mathbb{P}_{x}\left(X_{\tau_{M, N}^{A, B}}^{A, B}=M\right)<1$ and $0<\mathbb{P}_{x}\left(X_{\tau_{M, N}^{A, B}}^{A, B}=N\right)<1$;

(ii) $0<\mathbb{P}_{x}\left(\tau_{M}^{A, B}<\infty\right)<1$ and $0<\mathbb{P}_{x}\left(\tau_{N}^{A, B}<\infty\right)<1$.

Proof. We drop superscripts of the hitting times. We first prove (i). Note that it holds that $K\left(1 / 2 K \eta_{t}^{2}+\xi_{t}\right)>0$ for $t \in\left(0, \tau_{M, N}\right)$ for both positive and negative values of $K$ when $|K|$ is large. From (3.4), we have $\mathbb{E}_{x}\left(\exp \left\{K X_{\tau_{M, N}^{A, B}}^{A, T}\right\}\right)-\mathrm{e}^{K x} \geq 0$. Since $\mathbb{P}\left(\tau_{M, N}<\infty\right)=1$ (Lemma 3.1), letting $T \rightarrow \infty$, we have

$$
1 \leq \mathbb{E}_{x}\left[\exp \left\{K\left(X_{\tau_{M, N}}^{A, B}-x\right)\right\}\right]=\mathrm{e}^{K(M-x)} \mathbb{P}_{x}\left(X_{\tau_{M, N}}^{A, B}=M\right)+\mathrm{e}^{K(N-x)} \mathbb{P}_{x}\left(X_{\tau_{M, N}}^{A, B}=N\right) .
$$

Setting $K>0$ in the above inequality, we conclude that $\mathbb{P}_{x}\left(X_{\tau_{M, N}}^{A, B}=N\right)>0$, while setting $K<0$, we have $\mathbb{P}_{x}\left(X_{\tau_{M, N}}^{A, B}=M\right)>0$. From Lemma 3.1 we have $\mathbb{P}_{x}\left(X_{\tau_{M, N}}^{A, B}=M\right)+$ $\mathbb{P}_{x}\left(X_{\tau_{M, N}}^{A, B}=N\right)=1$; hence, the results in (i) hold.

Now we prove (ii). Note that $\mathbb{P}_{x}\left(\tau_{N}<\infty\right) \geq \mathbb{P}_{x}\left(X_{\tau_{M, N}}^{A, B}=N\right)>0$ (i.e. the probability of hitting $N$ is greater than the probability of hitting $N$ without hitting $M$ ). To show that $\mathbb{P}_{x}\left(\tau_{N}<\infty\right)<1$, we first consider the case when $N$ is negative and $|N|$ is large such that, for a fixed $K>0$, we have $K\left(1 / 2 K \eta_{t}^{2}+\xi_{t}\right)<0$ for $t \in\left(0, \tau_{M, N}\right)$. From (3.4) we have $\mathbb{E}_{x}\left(\exp \left\{K X_{\tau_{M, N} \wedge T}^{A, B}\right\}\right)-\mathrm{e}^{K x} \leq 0$. Hence,

$$
1 \geq \mathbb{E}_{x}\left[\exp \left\{K\left(X_{\tau_{M, N}}^{A, B}-x\right)\right\}\right]=\mathrm{e}^{K(M-x)} \mathbb{P}_{x}\left(X_{\tau_{M, N}}^{A, B}=M\right)+\mathrm{e}^{K(N-x)} \mathbb{P}_{x}\left(X_{\tau_{M, N}}^{A, B}=N\right) .
$$

By letting $M \rightarrow-\infty$ we obtain $1 \geq \mathrm{e}^{K(N-x)} \mathbb{P}_{x}\left(\tau_{N}<\infty\right)$, which yields $\mathbb{P}_{x}\left(\tau_{N}<\infty\right)<1$ or $\mathbb{P}_{x}\left(\tau_{N}=\infty\right)>0$. Now we prove the general case for any $x$ and $N$ with $x<N$. Take $x_{0}<N_{0}<x<N$ with $N_{0}<0$ and large $\left|N_{0}\right|$ such that $\mathbb{P}_{x_{0}}\left(\tau_{N_{0}}=\infty\right)>0$. Then

$$
1-\mathbb{P}_{x}\left(\tau_{N}<\infty\right)=\mathbb{P}_{x}\left(\tau_{N}=\infty\right) \geq \mathbb{P}_{x}\left(X_{\tau_{x_{0}, N}}^{A, B}=x_{0}\right) \mathbb{P}_{x_{0}}\left(\tau_{N_{0}}=\infty\right)>0 .
$$

The above inequality is due to the fact that the probability of the process never hitting $N$ is larger than the probability of the process first hitting $x_{0}$ without hitting $N$ and then never hitting $N_{0}$ afterwards. So we have shown that $0<\mathbb{P}_{x}\left(\tau_{N}<\infty\right)<1$. Likewise, we can show that $0<\mathbb{P}_{x}\left(\tau_{M}<\infty\right)<1$. 
We now show an ergodicity result of the controlled process.

Lemma 3.3. The controlled process $X^{A, B}$ governed by (2.1) under any admissible controls $A$ and $B$ diverges to $\infty$ or $-\infty$ almost surely.

Proof. In this proof we do not distinguish between sets that differ by a set having probability 0 . First we note that, for any $-\infty<M<N<\infty$,

$$
\mathbb{P}_{x}\left(\liminf _{t \rightarrow \infty} X_{t}^{A, B} \leq M \cap \limsup _{t \rightarrow \infty} X_{t}^{A, B} \geq N\right)=0 .
$$

In fact, the event $\liminf _{t \rightarrow \infty} X_{t}^{A, B} \leq M \cap \lim \sup _{t \rightarrow \infty} X_{t}^{A, B} \geq N$ implies that there are infinitely many crossing times of the interval $[M+\varepsilon, N-\varepsilon]$ for some $\varepsilon>0$. From Lemma 3.2, each time the process hits $M+\varepsilon$, there is a positive probability that the process never hits $N-\varepsilon$. Thus, the probability of having infinitely many crossing times is 0 and (3.5) holds. Letting $N \rightarrow M$ in (3.5), we obtain

$$
\mathbb{P}_{x}\left(\liminf _{t \rightarrow \infty} X_{t}^{A, B} \leq M \cap \limsup _{t \rightarrow \infty} X_{t}^{A, B}>M\right)=0 .
$$

This implies that

$$
\mathbb{P}_{x}\left(\liminf _{t \rightarrow \infty} X_{t}^{A, B} \leq M \cap \limsup _{t \rightarrow \infty} X_{t}^{A, B} \leq M\right)=\mathbb{P}_{x}\left(\liminf _{t \rightarrow \infty} X_{t}^{A, B} \leq M\right) .
$$

Thus, the events $\liminf _{t \rightarrow \infty} X_{t}^{A, B} \leq M$ and $\lim \sup _{t \rightarrow \infty} X_{t}^{A, B} \leq M$ are the same. Similarly, we can show that the events $\liminf _{t \rightarrow \infty} X_{t}^{A, B} \geq M$ and $\lim _{\sup _{t \rightarrow \infty}} X_{t}^{A, B} \geq M$ are the same.

We now show that

$$
\mathbb{P}_{x}\left(M \leq \lim \inf X_{t}^{A, B} \leq N\right)=0
$$

for any $-\infty<M<N<\infty$. Note that the event $M \leq \liminf X_{t}^{A, B} \leq N$ is the same as $M \leq \lim \sup X_{t}^{A, B} \leq N$. For any $\omega \in\left\{M \leq \lim \inf X_{t}^{A, B} \leq N\right\}$, we construct a sequence of hitting times as follows. If $x \in[M-\varepsilon / 2, N+\varepsilon / 2]$ for a fixed $\varepsilon>0$ then let $h_{1}=0$; otherwise, let $h_{1}$ be the first hitting time of the interval $[M-\varepsilon / 2, N+\varepsilon / 2]$ and note that we have $h_{1}<\infty$ by the definition of the event. Now let $h_{2}>h_{1}$ be the smallest time that the process exits $[M-\varepsilon, N+\varepsilon]$. From Lemma 3.1 we have $h_{2}<\infty$. Now we can define $h_{3}>h_{2}$ as the smallest hitting time of the interval $[M-\varepsilon / 2, N+\varepsilon / 2]$ after $h_{2}$, and note that $h_{3}<\infty$. Repeating the procedure, we obtain a sequence of hitting times with infinitely many times at $M-\varepsilon$ or $N+\varepsilon$ and also infinitely many times at $M-\varepsilon / 2$ or $N+\varepsilon / 2$. From Lemma 3.2, this happens with probability 0 and so (3.7) is proved.

Letting $M \rightarrow-\infty$ and $N \rightarrow \infty$ in (3.7), we have $\mathbb{P}_{x}\left(-\infty<\liminf X_{t}^{A, B}<\infty\right)=0$. Thus,

$$
\mathbb{P}_{x}\left(\liminf X_{t}^{A, B}=-\infty\right)+\mathbb{P}_{x}\left(\liminf X_{t}^{A, B}=\infty\right)=1 .
$$

Note that the events $\lim \inf X_{t}^{A, B}=\infty$ and $\lim X_{t}^{A, B}=\infty$ are the same. In addition, letting $M \rightarrow-\infty$ in (3.6), we see that the events $\lim \inf X_{t}^{A, B}=-\infty$ and $\lim X_{t}^{A, B}=-\infty$ are the same. This completes the proof.

In the following, we prove the verification theorem.

Theorem 3.1. Suppose that $W$ is a decreasing $C^{2}(-\infty, \infty)$ solution to FBI equations (3.1) subject to boundary conditions (3.3) and that $W^{\prime}$ is bounded. Then the function $W$ coincides 
with the value function $V$. Furthermore, the feedback control strategy $\left(A^{*}, B^{*}\right)$, determined by $A^{*}=\left\{a^{*}\left(X_{t}^{A^{*}, B^{*}}\right)\right\}_{t \geq 0}$ and $B^{*}=\left\{b^{*}\left(X_{t}^{A^{*}, B^{*}}\right)\right\}_{t \geq 0}$ with $a^{*}(x)$ and $b^{*}(x)$ given in (3.2), is the Nash equilibrium control such that $V^{A^{*}, B^{*}}(x)=V(x)$.

Proof. For any $-\infty<M<x<N<\infty$, admissible controls $A^{*}$ and $B$, and time $T>0$, by applying Itô's formula to $W\left(X_{t}^{A^{*}, B}\right)$ we obtain

$$
\begin{aligned}
W\left(X_{\tau_{M, N}^{A^{*}, B} \wedge T}^{A^{*}, B}\right) & W(x) \\
= & \int_{0}^{\tau_{M, N}^{A^{*}, B} \wedge T} L^{a^{*}\left(X_{t}^{A^{*}, B}\right), b_{t}} W\left(X_{t}^{A^{*}, B}\right) \mathrm{d} t+\sigma_{1} \int_{0}^{\tau_{M, N}^{A^{*}, B} \wedge T} W^{\prime}\left(X_{t}^{A^{*}, B}\right) a^{*}\left(X_{t}^{A^{*}, B}\right) \mathrm{d} w_{1} \\
& \quad-\sigma_{2} \int_{0}^{\tau_{M, N}^{A^{*}, B} \wedge T} W^{\prime}\left(X_{t}^{A^{*}, B}\right) b_{t} \mathrm{~d} w_{2}+\sigma \int_{0}^{\tau_{M, N}^{A^{*}, B} \wedge T} W^{\prime}\left(X_{t}^{A^{*}, B}\right) \mathrm{d} Z_{t} \\
\leq & \sigma_{1} \int_{0}^{\tau_{M, N}^{A^{*}, B} \wedge T} W^{\prime}\left(X_{t}^{A^{*}, B}\right) a^{*}\left(X_{t}^{A^{*}, B}\right) \mathrm{d} w_{1}-\sigma_{2} \int_{0}^{\tau_{M, N}^{A^{*}, B} \wedge T} W^{\prime}\left(X_{t}^{A^{*}, B}\right) b_{t} \mathrm{~d} w_{2} \\
& +\sigma \int_{0}^{\tau_{M, N}^{A^{*}, B} \wedge T} W^{\prime}\left(X_{t}^{A^{*}, B}\right) \mathrm{d} Z_{t},
\end{aligned}
$$

where the second line follows because $W$ solves the first equation in (3.1). Taking the expectation of both sides we obtain

$$
\begin{aligned}
W(x) \geq & \mathbb{E}_{x}\left[W\left(X_{\tau_{M, N}^{A^{*}, B} \wedge T}^{A^{*},}\right)\right] \\
= & \mathbb{P}_{x}\left(X_{\tau_{M, N}^{A^{*}, B}}^{A^{*}, B}=M, \tau_{M, N}^{A^{*}, B}<T\right) W(M)+\mathbb{P}_{x}\left(X_{\tau_{M, N}^{A^{*}, B}}^{A^{*}, B}=N, \tau_{M, N}^{A^{*}, B}<T\right) W(N) \\
& +\mathbb{E}_{x}\left[W\left(X_{T}^{A^{*}, B}\right) \mathbf{1}_{\left\{\tau_{M, N}^{A^{*}, B}>T\right\}}\right] .
\end{aligned}
$$

Letting $T \rightarrow \infty$, and noting that $\lim _{T \rightarrow \infty} \mathbb{P}\left(\tau_{M, N}^{A^{*}, B}>T\right)=0$ by Lemma 3.1 , we have

$$
W(x) \geq \mathbb{P}_{x}\left(X_{\tau_{M, N}^{A^{*}, B}}^{A^{*}, B}=M\right) W(M)+\mathbb{P}_{x}\left(X_{\tau_{M, N}^{A^{*}, B}}^{A^{*}, B}=N\right) W(N) .
$$

Letting $N \rightarrow \infty$, from the boundary conditions, we have

$$
W(x) \geq \mathbb{P}_{x}\left(X_{\tau_{M}^{A^{*}, B}}^{A^{*}, B}=M, \tau_{M}^{A^{*}, B}<\infty\right) W(M)=\mathbb{P}_{x}\left(\tau_{M}^{A^{*}, B}<\infty\right) W(M) .
$$

Note that

$$
V^{A^{*}, B}(x)=\mathbb{P}_{x}\left(\lim _{t \rightarrow \infty} X_{t}^{A^{*}, B}=-\infty\right)=\lim _{M \rightarrow-\infty} \mathbb{P}_{x}\left(\tau_{M}^{A^{*}, B}<\infty\right)
$$

Letting $M \rightarrow-\infty$ in (3.9), we obtain

$$
W(x) \geq V^{A^{*}, B}(x) .
$$

Similarly, we can show that

$$
W(x) \leq V^{A, B^{*}}(x) .
$$


Thus,

$$
\underline{V}(x) \geq \inf _{A} V^{A, B^{*}}(x) \geq W(x) \geq \sup _{B} V^{A^{*}, B}(x) \geq \bar{V}(x) .
$$

From $\underline{V}(x) \leq \bar{V}(x)$, we conclude that $\underline{V}(x)=W(x)=\bar{V}(x)=V(x)$.

Furthermore, if we replace $B$ by $B^{*}$ in (3.8), noting that

$$
L^{a^{*}\left(X_{t}^{A^{*}, B^{*}}\right), b^{*}\left(X_{t}^{A^{*}, B^{*}}\right)} W\left(X_{t}^{A^{*}, B^{*}}\right)=0,
$$

the first integrand on the right-hand side of (3.8) vanishes and the inequality becomes an equality. Consequently, inequality (3.9) becomes an equality. We then obtain

$$
W(x)=V^{A^{*}, B^{*}}(x) .
$$

Thus, the value function equals the performance function under the Nash equilibrium strategy $\left(A^{*}, B^{*}\right)$. This completes the proof.

Owing to the verification theorem, we call the game solvable if there exists a decreasing $C^{2}$ solution to (3.1) subject to boundary conditions (3.3) and the saddle-point strategy $\left(a^{*}(x), b^{*}(x)\right)$ given in (3.2).

Remark 3.1. From Lemma 3.3 we see that the definition of absolute dominance can be restated as the event that the difference in surplus levels tends to $-\infty$. Given any surplus difference $x$ and any paired admissible strategy $(A, B), V^{A, B}(x)$ is the probability of absolute dominance of insurer two and $1-V^{A, B}(x)$ is the probability of absolute dominance of insurer one.

Remark 3.2. From Lemmas 3.2 and 3.3, for any insurer at an arbitrary surplus difference level, there is always a positive probability of absolute dominance.

Remark 3.3. The performance function $V^{A, B}$ is a strictly decreasing function for any admissible controls $A$ and $B$. In fact, for any $x<y$, there is a positive probability, denoted by $p$ $(0<p<1)$, that the process $X^{A, B}$, starting at initial difference $x$, diverges to $-\infty$ without hitting $y$. Thus,

$$
V^{A, B}(x)=p+(1-p) V^{A, B}(y)=V^{A, B}(y)+p\left[1-V^{A, B}(y)\right]>V^{A, B}(y) .
$$

When the game is solvable, the value function $V$ is a strictly decreasing function.

\section{Solution to the FBI equations}

In this section we solve (3.1) and find the value function explicitly for the cases when the game is solvable. We characterize the solution via different regions where optimal reinsurance controls vary and the Nash equilibrium strategy takes different forms.

For any $C^{2}$ function $W$ with $W^{\prime \prime}(x) \neq 0$, write

$$
a_{W, b}(x)=-\frac{\gamma_{1}}{\sigma_{1}^{2}} \frac{W^{\prime}(x)}{W^{\prime \prime}(x)}+\rho \frac{\sigma_{2}}{\sigma_{1}} b, \quad b_{W, a}(x)=\frac{\gamma_{2}}{\sigma_{2}^{2}} \frac{W^{\prime}(x)}{W^{\prime \prime}(x)}+\rho \frac{\sigma_{1}}{\sigma_{2}} a,
$$

which satisfy

$$
\left.\frac{\mathrm{d} L^{a, b} W(x)}{\mathrm{d} a}\right|_{a=a_{W, b}(x)}=0,\left.\quad \frac{\mathrm{d} L^{a, b} W(x)}{\mathrm{d} b}\right|_{b=b_{W, a}(x)}=0 .
$$

These expressions are used to determine the minimizer and maximizer in (3.1) upon which the saddle point can be found. Note that $L^{a, b} V(x)$ is a quadratic function of $a$ and $b$, and the 
second-degree terms $\left(a^{2}\right.$ and $\left.b^{2}\right)$ in the function have the same sign. Thus, the saddle points of $L^{a, b} V(x)$ (if it exists) must occur on the boundary of the control region $[0,1] \times[0,1]$. To characterize the solution of the FBI equations, we first find all possible saddle-point strategies and identify the corresponding regions (of $x$ values) where these strategies apply. Then a simplified differential equation is derived in each region for the solution. Finally, a complete solution is constructed by smooth-fit techniques at the endpoints of the regions.

In this section we study the parameter case of nonnegative correlation. Note that in the case of negative correlation the game is solvable. The solution can be found by a similar method to that used in [9] and so we omit it. Now we assume that $\rho \geq 0$ and that $V$ solves the FBI equations. In the following we proceed to find the solution in explicit form.

Note that if $V^{\prime \prime}(x)>0, a_{V, b}(x) \geq 0$ for any $0 \leq b \leq 1$. So the minimizer in $a$ of $L^{a, b} V(x)$ is $a_{V, b}(x)$ or 1 . Obviously, the maximizer in $b$ is 0 or 1 . Define the following regions:

$$
\begin{aligned}
& \mathcal{R}_{1}=\left\{x \mid V^{\prime \prime}(x)>0,0<a_{V, 1}(x)<1, b_{V, a_{V, 1}(x)}(x)<\frac{1}{2}\right\}, \\
& \mathcal{R}_{2}=\left\{x \mid V^{\prime \prime}(x)>0, a_{V, 1}(x) \geq 1, b_{V, 1}(x)<\frac{1}{2}\right\}, \\
& \mathcal{R}_{3}=\left\{x \mid V^{\prime \prime}(x)>0,0<a_{V, 0}(x)<1, b_{V, a_{V, 0}(x)}(x) \geq \frac{1}{2}\right\}, \\
& \mathcal{R}_{4}=\left\{x \mid V^{\prime \prime}(x)>0, a_{V, 0}(x) \geq 1, b_{V, 1}(x) \geq \frac{1}{2}\right\} .
\end{aligned}
$$

Thus, the pair $\left(a_{V, 1}(x), 1\right)$ is a saddle point of the function $L^{(a, b)} V(x)$ on $[0,1] \times[0,1]$ for all $x \in \mathcal{R}_{1}$ and it holds that

$$
L^{a_{V, 1}(x), 1} V(x)=0 .
$$

Similarly, we have

$$
L^{1,1} V(x)=0,
$$

and the pair $(1,1)$ is a saddle point of $L^{(a, b)} V(x)$ for $x \in \mathcal{R}_{2}$. Furthermore, $V$ solves

$$
L^{a_{V, 0}(x), 0} V(x)=0,
$$

and the pair $\left(a_{V, 0}(x), 0\right)$ is a saddle point of $L^{(a, b)} V(x)$ for $x \in \mathcal{R}_{3}$. Similarly, $V$ solves

$$
L^{1,0} V(x)=0
$$

and the pair $(1,0)$ is a saddle point of $L^{(a, b)} V(x)$ for $x \in \mathcal{R}_{4}$.

For $V^{\prime \prime}(x)<0$, we have $b_{V, a}(x)>0$. The minimizer of $L^{a, b} V(x)$ in $a$ is 0 or 1 and the maximizer in $b$ is $b_{V, a}$ or 1 . Define

$$
\begin{aligned}
& \mathcal{R}_{5}=\left\{x \mid V^{\prime \prime}(x)<0, a_{V, b_{V, 1}(x)}(x)<\frac{1}{2}, 0<b_{V, 1}(x)<1\right\}, \\
& \mathcal{R}_{6}=\left\{x \mid V^{\prime \prime}(x)<0, a_{V, 1}(x)<\frac{1}{2}, b_{V, 1}(x) \geq 1\right\}, \\
& \mathcal{R}_{7}=\left\{x \mid V^{\prime \prime}(x)<0, a_{V, b_{V, 0}(x)}(x) \geq \frac{1}{2}, 0<b_{V, 0}(x)<1\right\}, \\
& \mathcal{R}_{8}=\left\{x \mid V^{\prime \prime}(x)<0, a_{V, 1}(x) \geq \frac{1}{2}, b_{V, 0}(x) \geq 1\right\} .
\end{aligned}
$$

Then it holds that

$$
L^{1, b_{V, 1}(x)} V(x)=0,
$$

and the pair $\left(1, b_{V, 1}(x)\right)$ is a saddle point of the function $L^{(a, b)} V(x)$ for $x \in \mathcal{R}_{5}$. Furthermore, $V$ solves $(4.3)$ and $(1,1)$ is a saddle point of the function $L^{(a, b)} V(x)$ for $x \in \mathcal{R}_{6}$. It holds that

$$
L^{0, b_{V, 0}(x)} V(x)=0,
$$


and the pair $\left(1, b_{V, 1}(x)\right)$ is a saddle point of $L^{(a, b)} V(x)$ on $\mathcal{R}_{7}$. Finally, $V$ solves

$$
L^{0,1} V(x)=0
$$

and $(0,1)$ is a saddle point of $L^{(a, b)} V(x)$ on $\mathcal{R}_{8}$.

Now we identify the sets $\mathcal{R}_{1}, \ldots, \mathcal{R}_{8}$. To find $R_{1}$, we simplify (4.2) to

$$
\frac{\gamma_{1}^{2}}{2 \sigma_{1}^{2}}\left(\frac{V^{\prime}(x)}{V^{\prime \prime}(x)}\right)^{2}-\left[r x-\left(\mu_{2}+\lambda_{1}\right)+\gamma_{1} \rho \frac{\sigma_{2}}{\sigma_{1}}\right] \frac{V^{\prime}(x)}{V^{\prime \prime}(x)}-\frac{1}{2}\left[\left(1-\rho^{2}\right) \sigma_{2}^{2}+\sigma^{2}\right]=0,
$$

from which $V$ solves

$$
\begin{aligned}
\frac{V^{\prime}(x)}{V^{\prime \prime}(x)}= & f_{1}(x) \\
:= & \left\{r x-\left(\mu_{2}+\lambda_{1}\right)+\gamma_{1} \rho \frac{\sigma_{2}}{\sigma_{1}}\right. \\
& \left.\quad-\sqrt{\left[r x-\left(\mu_{2}+\lambda_{1}\right)+\gamma_{1} \rho \frac{\sigma_{2}}{\sigma_{1}}\right]^{2}+\frac{\gamma_{1}^{2}}{\sigma_{1}^{2}}\left[\left(1-\rho^{2}\right) \sigma_{2}^{2}+\sigma^{2}\right]}\right] / \frac{\gamma_{1}^{2}}{\sigma_{1}^{2}}
\end{aligned}
$$

for $x \in \mathcal{R}_{1}$. Note that $f_{1}$ is a negative increasing function. Also, note that

$$
a_{V, 1}(x)=-\frac{\gamma_{1}}{\sigma_{1}^{2}} f_{1}(x)+\rho \frac{\sigma_{2}}{\sigma_{1}}, \quad b_{V, a_{V, 1}(x)}=\kappa_{1} f_{1}(x)+\rho^{2},
$$

where $\kappa_{1}=\gamma_{2} / \sigma_{2}^{2}-\rho \gamma_{1} / \sigma_{1} \sigma_{2}$. From $a_{V, 1}(x)<1$ and $b_{V, a_{V, 1}(x)}(x)<\frac{1}{2}$, we have

$$
\mathcal{R}_{1}=\left\{x \mid-\left(1-\rho \frac{\sigma_{2}}{\sigma_{1}}\right) \frac{\sigma_{1}^{2}}{\gamma_{1}}<f_{1}(x)<\frac{1 / 2-\rho^{2}}{\kappa_{1}}\right\}
$$

if $\kappa_{1}>0$ (or $\left.\rho<\gamma_{2} \sigma_{1} / \gamma_{1} \sigma_{2}\right)$, and

$$
\mathcal{R}_{1}=\left\{x \mid \max \left\{-\left(1-\rho \frac{\sigma_{2}}{\sigma_{1}}\right) \frac{\sigma_{1}^{2}}{\gamma_{1}}, \frac{1 / 2-\rho^{2}}{\kappa_{1}}\right\}<f_{1}(x)\right\}
$$

if $\kappa_{1}<0$. The set $\mathcal{R}_{1}$ is degenerate $\left(\mathcal{R}_{1}=\varnothing\right)$ when $\rho \geq \sigma_{1} / \sigma_{2}$.

To determine $\mathcal{R}_{2}$, we note that (4.3) gives

$$
\frac{V^{\prime}(x)}{V^{\prime \prime}(x)}=f_{2}(x):=-\frac{\sigma_{1}^{2}+\sigma_{2}^{2}-2 \rho \sigma_{1} \sigma_{2}+\sigma^{2}}{2\left(r x+\mu_{1}-\mu_{2}\right)} .
$$

Thus, the conditions $V^{\prime \prime}(x)>0, a_{V, 1}(x) \geq 1$, and $b_{V, 1}(x)<\frac{1}{2}$ yield

$$
\begin{aligned}
\mathcal{R}_{2}=\{x \mid & \left(r x+\mu_{1}-\mu_{2}\right)>0 \\
& \left(1-\rho \frac{\sigma_{2}}{\sigma_{1}}\right)\left(r x+\mu_{1}-\mu_{2}\right) \leq \frac{\gamma_{1}\left(\sigma_{1}^{2}+\sigma_{2}^{2}-2 \rho \sigma_{1} \sigma_{2}+\sigma^{2}\right)}{2 \sigma_{1}^{2}} ; \\
& \left.\left(\frac{1}{2}-\rho \frac{\sigma_{1}}{\sigma_{2}}\right)\left(r x+\mu_{1}-\mu_{2}\right)>-\frac{\gamma_{2}\left(\sigma_{1}^{2}+\sigma_{2}^{2}-2 \rho \sigma_{1} \sigma_{2}+\sigma^{2}\right)}{2 \sigma_{2}^{2}}\right\} .
\end{aligned}
$$


For $x \in \mathcal{R}_{3}$, from (4.4), $V$ solves

$$
\frac{\gamma_{1}^{2}}{2 \sigma_{1}^{2}}\left(\frac{V^{\prime}(x)}{V^{\prime \prime}(x)}\right)^{2}-\left[r x-\left(\lambda_{1}-\lambda_{2}\right)\right] \frac{V^{\prime}(x)}{V^{\prime \prime}(x)}-\frac{1}{2} \sigma^{2}=0 .
$$

This gives

$$
\frac{V^{\prime}(x)}{V^{\prime \prime}(x)}=f_{3}(x):=\frac{r x-\left(\lambda_{1}-\lambda_{2}\right)-\sqrt{\left[r x-\left(\lambda_{1}-\lambda_{2}\right)\right]^{2}+\gamma_{1}^{2} \sigma^{2} / \sigma_{1}^{2}}}{\gamma_{1}^{2} / \sigma_{1}^{2}},
$$

where $f_{3}$ is a negative increasing function. The conditions $a_{V, 0}(x)<1$ and $b_{V, a_{V, 0}}(x) \geq \frac{1}{2}$ give

$$
\mathcal{R}_{3}=\left\{x \mid-\frac{\sigma_{1}^{2}}{\gamma_{1}}<f_{3}(x) \leq \frac{1}{2 \kappa_{1}}\right\}
$$

if $\kappa_{1}<0$ (or $\rho>\gamma_{2} \sigma_{1} / \gamma_{1} \sigma_{2}$ ). We can show that $\mathcal{R}_{3}$ is a nonempty interval if $\rho>\gamma_{2} \sigma_{1} / \gamma_{1} \sigma_{2}+$ $\frac{1}{2} \sigma_{2} / \sigma_{1}$ and degenerate otherwise.

For $x \in \mathcal{R}_{4}$, from (4.5), $V$ solves

$$
\frac{V^{\prime}(x)}{V^{\prime \prime}(x)}=f_{4}(x):=-\frac{\sigma_{1}^{2}+\sigma^{2}}{2\left(r x+\mu_{1}+\lambda_{2}\right)} .
$$

Then the conditions $V^{\prime \prime}(x)>0, a_{V, 0} \geq 1$, and $b_{V, 1}(x) \geq \frac{1}{2}$ give

$$
\begin{aligned}
& \mathcal{R}_{4}=\left\{x \mid 0<r x+\mu_{1}+\lambda_{2} \leq \frac{\gamma_{1}}{2}\left(1+\frac{\sigma^{2}}{\sigma_{1}^{2}}\right) ;\right. \\
& \left.\left(\frac{1}{2}-\rho \frac{\sigma_{1}}{\sigma_{2}}\right)\left(r x+\mu_{1}+\lambda_{2}\right) \leq-\frac{\gamma_{2}}{2} \frac{\sigma_{1}^{2}+\sigma^{2}}{\sigma_{2}^{2}}\right\},
\end{aligned}
$$

which is a nonempty interval if $\rho>\gamma_{2} \sigma_{1} / \gamma_{1} \sigma_{2}+\frac{1}{2} \sigma_{2} / \sigma_{1}$ and degenerate otherwise.

To identify $\mathcal{R}_{5}$, from (4.7), $V$ solves

$$
\frac{\gamma_{2}^{2}}{2 \sigma_{2}^{2}}\left(\frac{V^{\prime}(x)}{V^{\prime \prime}(x)}\right)^{2}-\left(r x+\mu_{1}+\lambda_{2}-\gamma_{2} \rho \frac{\sigma_{1}}{\sigma_{2}}\right) \frac{V^{\prime}(x)}{V^{\prime \prime}(x)}-\frac{1}{2}\left[\left(1-\rho^{2}\right) \sigma_{1}^{2}+\sigma^{2}\right]=0 .
$$

Thus,

$$
\begin{aligned}
\frac{V^{\prime}(x)}{V^{\prime \prime}(x)}= & f_{5}(x) \\
:= & \left\{r x+\mu_{1}+\lambda_{2}-\gamma_{2} \rho \frac{\sigma_{1}}{\sigma_{2}}\right. \\
& \left.\quad+\sqrt{\left(r x+\mu_{1}+\lambda_{2}-\gamma_{2} \rho \frac{\sigma_{1}}{\sigma_{2}}\right)^{2}+\frac{\gamma_{2}^{2}}{\sigma_{2}^{2}}\left[\left(1-\rho^{2}\right) \sigma_{1}^{2}+\sigma^{2}\right]}\right\} / \frac{\gamma_{2}^{2}}{\sigma_{2}^{2}},
\end{aligned}
$$

which is a positive and increasing function. The conditions $a_{V, b_{V, 1}(x)}(x)<\frac{1}{2}$ and $b_{V, 1}(x)<1$ give

$$
\mathcal{R}_{5}=\left\{x \mid \frac{1 / 2-\rho^{2}}{\kappa_{2}}<f_{5}(x)<\left(1-\rho \frac{\sigma_{1}}{\sigma_{2}}\right) \frac{\sigma_{2}^{2}}{\gamma_{2}}\right\}
$$


if $\kappa_{2}<0$ (or $\left.\rho<\gamma_{1} \sigma_{2} / \gamma_{2} \sigma_{1}\right)$, where $\kappa_{2}=-\gamma_{1} / \sigma_{1}^{2}+\rho \gamma_{2} / \sigma_{1} \sigma_{2}$, and

$$
\mathcal{R}_{5}=\left\{x \mid f_{5}(x)<\min \left\{\frac{1 / 2-\rho^{2}}{\kappa_{2}},\left(1-\rho \frac{\sigma_{1}}{\sigma_{2}}\right) \frac{\sigma_{2}^{2}}{\gamma_{2}}\right\}\right\}
$$

if $\kappa_{2}>0$. Note that $\mathcal{R}_{5}$ is degenerate if $\rho \geq \sigma_{2} / \sigma_{1}$.

For $x \in \mathcal{R}_{6}, V$ solves (4.3) and it holds that $V^{\prime}(x) / V^{\prime \prime}(x)=f_{2}(x)$. Thus, the conditions $V^{\prime \prime}(x)<0, a_{V, 1}(x)<\frac{1}{2}$, and $b_{V, 1}(x) \geq 1$ give

$$
\begin{aligned}
& \mathcal{R}_{6}:=\left\{x \mid r x+\mu_{1}-\mu_{2}<0 ;\right. \\
&\left(\frac{1}{2}-\rho \frac{\sigma_{2}}{\sigma_{1}}\right)\left(r x+\mu_{1}-\mu_{2}\right)<\frac{\gamma_{1}\left(\sigma_{1}^{2}+\sigma_{2}^{2}-2 \rho \sigma_{1} \sigma_{2}+\sigma^{2}\right)}{2 \sigma_{1}^{2}} ; \\
&\left.\left(1-\rho \frac{\sigma_{1}}{\sigma_{2}}\right)\left(r x+\mu_{1}-\mu_{2}\right) \geq-\frac{\gamma_{2}\left(\sigma_{1}^{2}+\sigma_{2}^{2}-2 \rho \sigma_{1} \sigma_{2}+\sigma^{2}\right)}{2 \sigma_{2}^{2}}\right\} .
\end{aligned}
$$

For $x \in \mathcal{R}_{7}$, from (4.8), $V$ solves

$$
\frac{V^{\prime}(x)}{V^{\prime \prime}(x)}=f_{7}(x):=\frac{r x-\left(\lambda_{1}-\lambda_{2}\right)+\sqrt{\left[r x-\left(\lambda_{1}-\lambda_{2}\right)\right]^{2}+\gamma_{2}^{2} \sigma^{2} / \sigma_{2}^{2}}}{\gamma_{2}^{2} / \sigma_{2}^{2}} .
$$

The conditions $a_{V, b_{V, 0}(x)}(x) \geq \frac{1}{2}$ and $b_{V, 0}(x)<1$ give

$$
\mathcal{R}_{7}=\left\{x \mid \frac{1}{2 \kappa_{2}} \leq f_{7}(x)<\frac{\sigma_{2}^{2}}{\gamma_{2}}\right\}
$$

if $\kappa_{2}>0$ (or $\rho>\gamma_{1} \sigma_{2} / \gamma_{2} \sigma_{1}$ ). Note that $\mathcal{R}_{7}$ is a nonempty interval if $\rho>\frac{1}{2} \sigma_{1} / \sigma_{2}+\gamma_{1} \sigma_{2} / \gamma_{2} \sigma_{1}$ and degenerate otherwise.

For $x \in \mathcal{R}_{8}$, from (4.9), $V$ solves

$$
\frac{V^{\prime}(x)}{V^{\prime \prime}(x)}=f_{8}(x):=-\frac{\sigma_{2}^{2}+\sigma^{2}}{2\left[r x-\left(\mu_{2}+\lambda_{1}\right)\right]} .
$$

Thus, the conditions $V^{\prime \prime}(x)<0, a_{V, 1}(x) \geq \frac{1}{2}$, and $b_{V, 0}(x) \geq 1$ give

$$
\mathcal{R}_{8}=\left\{x \mid r x-\left(\mu_{2}+\lambda_{1}\right)<0 ;-\frac{\left(\sigma_{2}^{2}+\sigma^{2}\right) \gamma_{2}}{2 \sigma_{2}^{2}} \leq r x-\left(\mu_{2}+\lambda_{1}\right) \leq \frac{\left(\sigma_{2}^{2}+\sigma^{2}\right) \gamma_{1} / \sigma_{1}^{2}}{1-2 \rho \sigma_{2} / \sigma_{1}}\right\},
$$

when $\rho>\frac{1}{2} \sigma_{1} / \sigma_{2}$. Note that $\mathcal{R}_{8}$ is a nonempty interval if $\rho>\frac{1}{2} \sigma_{1} / \sigma_{2}+\gamma_{1} \sigma_{2} / \gamma_{2} \sigma_{1}$ and degenerate otherwise.

We define the threshold points

$$
\begin{gathered}
x_{1}=x_{2}+\frac{\gamma_{1}\left(\sigma_{1}^{2}+\sigma_{2}^{2}-2 \rho \sigma_{1} \sigma_{2}+\sigma^{2}\right)}{2 r \sigma_{1}^{2}\left(1-\rho \sigma_{2} / \sigma_{1}\right)}, \quad x_{2}=-\frac{\mu_{1}-\mu_{2}}{r}, \\
\text { and } x_{3}=x_{2}-\frac{\gamma_{2}\left(\sigma_{1}^{2}+\sigma_{2}^{2}-2 \rho \sigma_{1} \sigma_{2}+\sigma^{2}\right)}{2 r \sigma_{2}^{2}\left(1-\rho \sigma_{1} / \sigma_{2}\right)},
\end{gathered}
$$


and write

$$
\zeta_{1}=\left(\frac{1}{2}+\frac{\gamma_{2}}{\gamma_{1}} \frac{\sigma_{1}^{2}}{\sigma_{2}^{2}}\right) /\left(\frac{\gamma_{2}}{\gamma_{1}} \frac{\sigma_{1}}{\sigma_{2}}+\frac{\sigma_{1}}{\sigma_{2}}\right), \quad \zeta_{2}=\left(\frac{1}{2}+\frac{\gamma_{1}}{\gamma_{2}} \frac{\sigma_{2}^{2}}{\sigma_{1}^{2}}\right) /\left(\frac{\gamma_{1}}{\gamma_{2}} \frac{\sigma_{2}}{\sigma_{1}}+\frac{\sigma_{2}}{\sigma_{1}}\right) .
$$

We obtain the following properties.

Lemma 4.1. The region $\mathcal{R}_{1}$ defined in (4.1a) is such that $\mathcal{R}_{1}=\left\{x \mid x_{1}<x<\infty\right\}$ if and only if $\rho<\min \left\{1 / \sqrt{2}, \sigma_{1} / \sigma_{2}, \gamma_{2} \sigma_{1} / \gamma_{1} \sigma_{2}\right\}$ or $\gamma_{2} \sigma_{1} / \gamma_{1} \sigma_{2}<\rho<\min \left\{\sigma_{1} / \sigma_{2}, \zeta_{1}\right\}$.

Lemma 4.2. The region $\mathcal{R}_{2}$ defined in (4.1b) is such that $\mathcal{R}_{2}=\left\{x \mid x_{2}<x \leq x_{1}\right\}$ if and only if $\rho<\min \left\{\sigma_{1} / \sigma_{2}, \frac{1}{2} \sigma_{2} / \sigma_{1}\right\}$ or $\frac{1}{2} \sigma_{2} / \sigma_{1} \leq \rho<\min \left\{\sigma_{1} / \sigma_{2}, \zeta_{1}\right\}$.

Lemma 4.3. The region $\mathcal{R}_{5}$ defined in (4.6a) is such that $\mathcal{R}_{5}=\left\{x \mid-\infty<x<x_{3}\right\}$ if and only if $\rho<\min \left\{1 / \sqrt{2}, \sigma_{2} / \sigma_{1}, \gamma_{1} \sigma_{2} / \gamma_{2} \sigma_{1}\right\}$ or $\gamma_{1} \sigma_{2} / \gamma_{2} \sigma_{1}<\rho<\min \left\{\sigma_{2} / \sigma_{1}, \zeta_{2}\right\}$.

Lemma 4.4. The region $\mathcal{R}_{6}$ defined in (4.6b) is such that $\mathcal{R}_{6}=\left\{x \mid x_{3} \leq x<x_{2}\right\}$ if and only if $\rho<\min \left\{\sigma_{2} / \sigma_{1}, \frac{1}{2} \sigma_{1} / \sigma_{2}\right\}$ or $\frac{1}{2} \sigma_{1} / \sigma_{2} \leq \rho<\min \left\{\sigma_{2} / \sigma_{1}, \zeta_{2}\right\}$.

Define

$$
\mathcal{R}_{0}=\left\{x \mid V^{\prime \prime}(x)=0\right\} .
$$

Remark 4.1. If $V^{\prime \prime}(x)=0$ and $V^{\prime}(x)<0$, the saddle point of $L^{a, b} V(x)$ is $(1,1)$. Thus, $V$ solves $L^{1,1} V(x)=0$ and it gives $x=x_{2}$. Hence,

$$
\mathcal{R}_{0}=\left\{x \mid V^{\prime \prime}(x)=0, V^{\prime}(x)=0\right\} \cup\left\{x_{2}\right\},
$$

from which we see that $R_{0}$ never contains an interval because $V$ is a strictly decreasing function (see Remark 3.3).

Next we consider the case with small correlation $\rho$ under which the sets $\mathcal{R}_{1}, \mathcal{R}_{2}, \mathcal{R}_{5}$, and $\mathcal{R}_{6}$ form a partition of the real line, excluding $x_{2}$, and an explicit solution of $V$ can be constructed. Suppose that $\rho<\min \left\{\frac{1}{2} \sigma_{1} / \sigma_{2}, \frac{1}{2} \sigma_{2} / \sigma_{1}, \gamma_{2} \sigma_{1} / \gamma_{1} \sigma_{2}, \gamma_{1} \sigma_{2} / \gamma_{2} \sigma_{1}\right\}$. Note that $\rho<\frac{1}{2}$ under the assumption. From Lemmas 4.1-4.4 we have

$$
\begin{gathered}
\mathcal{R}_{3}=\mathcal{R}_{4}=\mathcal{R}_{7}=\mathcal{R}_{8}=\varnothing, \\
\mathcal{R}_{1}=\left\{x \mid x_{1}<x<\infty\right\}, \quad \mathcal{R}_{2}=\left\{x \mid x_{2}<x \leq x_{1}\right\}, \\
\mathcal{R}_{6}=\left\{x \mid x_{3} \leq x<x_{2}\right\}, \quad \mathcal{R}_{5}=\left\{x \mid-\infty<x<x_{3}\right\} .
\end{gathered}
$$

Thus, $V$ solves

$$
\frac{V^{\prime}(x)}{V^{\prime \prime}(x)}= \begin{cases}f_{1}(x), & \left\{x_{1}<x<\infty\right\}=\mathcal{R}_{1} \\ f_{2}(x), & \left\{x_{3} \leq x \leq x_{1}\right\}=\mathcal{R}_{2} \cup \mathcal{R}_{6} \cup\left\{x_{2}\right\} \\ f_{5}(x), & \left\{-\infty<x<x_{3}\right\}=\mathcal{R}_{5}\end{cases}
$$

By applying the boundary conditions (3.3) and smooth-fit conditions at the threshold points $x_{1}$ and $x_{3}$,

$$
\begin{array}{ll}
V\left(x_{1}-\right)=V\left(x_{1}+\right), & V^{\prime}\left(x_{1}-\right)=V^{\prime}\left(x_{1}+\right), \\
V\left(x_{3}-\right)=V\left(x_{3}+\right), & V^{\prime}\left(x_{3}-\right)=V^{\prime}\left(x_{3}+\right),
\end{array}
$$


we can obtain the explicit representation of $V$. Write

$$
\begin{gathered}
G_{1}(y, x)=\int_{x}^{\infty} \exp \left\{\int_{y}^{u} \frac{1}{f_{1}(v)} \mathrm{d} v\right\} \mathrm{d} u, \quad F_{2}(y, x)=\int_{y}^{x} \exp \left\{\int_{y}^{u} \frac{1}{f_{2}(v)} \mathrm{d} v\right\} \mathrm{d} u, \\
H_{5}(y, x)=\int_{-\infty}^{x} \exp \left\{\int_{y}^{u} \frac{1}{f_{5}(v)} \mathrm{d} v\right\} \mathrm{d} u .
\end{gathered}
$$

We obtain the following theorem.

Theorem 4.1. If $\leq \rho \rho \min \left\{\frac{1}{2} \sigma_{1} / \sigma_{2}, \frac{1}{2} \sigma_{2} / \sigma_{1}, \gamma_{2} \sigma_{1} / \gamma_{1} \sigma_{2}, \gamma_{1} \sigma_{2} / \gamma_{2} \sigma_{1}\right\}$, the value function of the game is a decreasing $C^{2}$ function given by

$$
V(x)= \begin{cases}\frac{C_{2} G_{1}\left(x_{1}, x\right)}{C_{1}}, & x_{1}<x<\infty, \\ 1-\frac{H_{5}\left(x_{3}, x_{3}\right)}{C_{1}}-\frac{F_{2}\left(x_{3}, x\right)}{C_{1}}, & x_{3}<x \leq x_{1}, \\ 1-\frac{H_{5}\left(x_{3}, x\right)}{C_{1}}, & -\infty<x \leq x_{3},\end{cases}
$$

where $C_{1}=C_{2} G_{1}\left(x_{1}, x_{1}\right)+F_{2}\left(x_{3}, x_{1}\right)+H_{5}\left(x_{3}, x_{3}\right), C_{2}=\exp \left\{\int_{x_{3}}^{x_{1}}\left(1 / f_{2}(u)\right) \mathrm{d} u\right\}$, and the Nash equilibrium strategy is the feedback control associated with the risk exposure functions

$$
\left(a^{*}(x), b^{*}(x)\right)= \begin{cases}\left(-\frac{\gamma_{1}}{\sigma_{1}^{2}} f_{1}(x)+\rho \frac{\sigma_{2}}{\sigma_{1}}, 1\right), & x_{1}<x<\infty, \\ (1,1), & x_{3}<x \leq x_{1}, \\ \left(1, \frac{\gamma_{2}}{\sigma_{2}^{2}} f_{5}(x)+\rho \frac{\sigma_{1}}{\sigma_{2}}\right), & -\infty<x \leq x_{3} .\end{cases}
$$

Proof. From the construction of $V$ we can check that $V$ is a decreasing $C^{2}$ function and it solves the FBI equations (3.1) subject to boundary conditions (3.3). The saddle-point strategy given in (4.11) satisfies (3.2) and $V^{\prime}$ is bounded. Thus, the results follow from the verification theorem, Theorem 3.1 .

Remark 4.2. In Theorem 4.1 the risk exposure functions $a^{*}(x)$ and $b^{*}(x)$ are continuous and monotone. The function $a^{*}(x)$ is decreasing and $\lim _{x \rightarrow \infty} a^{*}(x)=\rho \sigma_{2} / \sigma_{1}$. The function $b^{*}(x)$ is increasing and $\lim _{x \rightarrow-\infty} b^{*}(x)=\rho \sigma_{1} / \sigma_{2}$. This implies that the Nash equilibrium strategy has the following property. When the surplus difference increases (insurer one is into a better position), insurer one takes less risk and buys more reinsurance while insurer two takes more risk and buys less reinsurance.

Remark 4.3. Under the parameter assumption

$$
\min \left\{\frac{\gamma_{2}}{\gamma_{1}} \frac{\sigma_{1}}{\sigma_{2}}, \frac{\gamma_{1}}{\gamma_{2}} \frac{\sigma_{2}}{\sigma_{1}}\right\}<\rho<\min \left\{\frac{1}{\sqrt{2}}, \frac{\sigma_{1}}{\sigma_{2}}, \frac{\sigma_{2}}{\sigma_{1}}, \zeta_{1}, \zeta_{2}\right\},
$$

(4.10) holds. In fact, we can identify all of the parameter cases under which (4.10) holds by Lemmas 4.1-4.4. Consequently, the results in Theorem 4.1 also hold in the following cases.

Lemma 4.5. The region $\mathcal{R}_{2}$ defined in (4.1b) is such that $\mathcal{R}_{2}=\left\{x \mid x_{2}<x<\infty\right\}$ if and only if $\sigma_{1} / \sigma_{2} \leq \rho<\frac{1}{2} \sigma_{2} / \sigma_{1}$.

Lemma 4.6. The region $\mathcal{R}_{6}$ defined in (4.6b) is such that $\mathcal{R}_{6}=\left\{x \mid-\infty<x<x_{2}\right\}$ if and only if $\sigma_{2} / \sigma_{1} \leq \rho<\frac{1}{2} \sigma_{1} / \sigma_{2}$. 
Now consider the parameter condition $\sigma_{1} / \sigma_{2} \leq \rho<\min \left\{1 / \sqrt{2}, \frac{1}{2} \sigma_{2} / \sigma_{1}, \gamma_{1} \sigma_{2} / \gamma_{2} \sigma_{1}, \zeta_{2}\right\}$, under which we see that

$$
\begin{gathered}
\mathcal{R}_{1}=\mathcal{R}_{3}=\mathcal{R}_{4}=\mathcal{R}_{7}=\mathcal{R}_{8}=\varnothing, \\
\mathcal{R}_{2}=\left\{x \mid x_{2}<x<\infty\right\}, \quad \mathcal{R}_{6}=\left\{x \mid x_{3} \leq x<x_{2}\right\}, \\
\mathcal{R}_{5}=\left\{x \mid-\infty<x<x_{3}\right\}
\end{gathered}
$$

Thus, $V$ solves

$$
\frac{V^{\prime}(x)}{V^{\prime \prime}(x)}= \begin{cases}f_{2}(x), & \left\{x_{3} \leq x \leq \infty\right\}=\mathcal{R}_{2} \cup \mathcal{R}_{6} \cup\left\{x_{2}\right\} \\ f_{5}(x), & \left\{-\infty<x<x_{3}\right\}=\mathcal{R}_{5}\end{cases}
$$

Define $G_{2}(y, x)=\int_{x}^{\infty} \exp \left\{\int_{y}^{u}\left(1 / f_{2}(v)\right) \mathrm{d} v\right\} \mathrm{d} u$. It holds that

$$
G_{2}(y, x)=\sqrt{\frac{2 \pi}{K_{1}}} \exp \left\{\frac{1}{2}\left(\sqrt{K_{1}} y+\frac{K_{2}}{\sqrt{K_{1}}}\right)^{2}\right\}\left[1-\Phi\left(\sqrt{K_{1}} x+\frac{K_{2}}{\sqrt{K_{1}}}\right)\right],
$$

where $\Phi$ is the standard normal distribution function and

$$
K_{1}=\frac{2 r}{\sigma_{1}^{2}+\sigma_{2}^{2}-2 \rho \sigma_{1} \sigma_{2}+\sigma^{2}}, \quad K_{2}=\frac{2\left(\mu_{1}-\mu_{2}\right)}{\sigma_{1}^{2}+\sigma_{2}^{2}-2 \rho \sigma_{1} \sigma_{2}+\sigma^{2}} .
$$

We obtain the following theorem.

Theorem 4.2. If $\sigma_{1} / \sigma_{2} \leq \rho<\min \left\{1 / \sqrt{2}, \frac{1}{2} \sigma_{2} / \sigma_{1}, \gamma_{1} \sigma_{2} / \gamma_{2} \sigma_{1}, \zeta_{2}\right\}$, the value function of the game is a decreasing $C^{2}$ function given by

$$
V(x)= \begin{cases}\frac{G_{2}\left(x_{3}, x\right)}{C_{3}}, & x_{3}<x<\infty, \\ 1-\frac{H_{5}\left(x_{3}, x\right)}{C_{3}}, & -\infty<x \leq x_{3},\end{cases}
$$

where $C_{3}=G_{2}\left(x_{3}, x_{3}\right)+H_{5}\left(x_{3}, x_{3}\right)$, and the Nash equilibrium strategy is the feedback control associated with the risk exposure functions

$$
\left(a^{*}(x), b^{*}(x)\right)= \begin{cases}(1,1), & x_{3}<x<\infty, \\ \left(1, \frac{\gamma_{2}}{\sigma_{2}^{2}} f_{5}(x)+\rho \frac{\sigma_{1}}{\sigma_{2}}\right), & -\infty<x \leq x_{3} .\end{cases}
$$

Remark 4.4. The results in (4.12) and Theorem 4.2 also hold under parameter condition $\max \left\{\sigma_{1} / \sigma_{2}, \gamma_{1} \sigma_{2} / \gamma_{2} \sigma_{1}\right\}<\rho<\min \left\{\frac{1}{2} \sigma_{2} / \sigma_{1}, \zeta_{2}\right\}$. The parameter conditions under which the results in Theorem 4.2 hold can be found by Lemmas 4.3, 4.4, and 4.5.

In the sequel we solve the game under the parameter conditions that mirror those in Theorem 4.2 and Remark 4.4. If $\sigma_{2} / \sigma_{1} \leq \rho<\min \left\{1 / \sqrt{2}, \frac{1}{2} \sigma_{1} / \sigma_{2}, \gamma_{2} \sigma_{1} / \gamma_{1} \sigma_{2}, \zeta_{1}\right\}$ then

$$
\begin{gathered}
\mathcal{R}_{3}=\mathcal{R}_{4}=\mathcal{R}_{5}=\mathcal{R}_{7}=\mathcal{R}_{8}=\varnothing, \\
\mathcal{R}_{1}=\left\{x \mid x_{1}<x<\infty\right\}, \quad \mathcal{R}_{2}=\left\{x \mid x_{2}<x \leq x_{1}\right\}, \\
\mathcal{R}_{6}=\left\{x \mid-\infty<x<x_{2}\right\} .
\end{gathered}
$$


Thus, $V$ solves

$$
\frac{V^{\prime}(x)}{V^{\prime \prime}(x)}= \begin{cases}f_{1}(x), & \left\{x_{1}<x<\infty\right\}=R_{1} \\ f_{2}(x), & \left\{x_{3} \leq x \leq x_{1}\right\}=R_{2} \cup R_{6} \cup\left\{x_{2}\right\}\end{cases}
$$

Write

$$
H_{2}(y, x)=\int_{-\infty}^{x} \exp \left\{\int_{y}^{u} \frac{1}{f_{2}(v)} \mathrm{d} v\right\} \mathrm{d} u
$$

and note that

$$
H_{2}(y, x)=\sqrt{\frac{2 \pi}{K_{1}}} \exp \left\{\frac{1}{2}\left(\sqrt{K_{1}} y+\frac{K_{2}}{\sqrt{K_{1}}}\right)^{2}\right\} \Phi\left(\sqrt{K_{1}} x+\frac{K_{2}}{\sqrt{K_{1}}}\right) .
$$

We have obtained the following theorem.

Theorem 4.3. If $\sigma_{2} / \sigma_{1} \leq \rho<\min \left\{1 / \sqrt{2}, \frac{1}{2} \sigma_{1} / \sigma_{2}, \gamma_{2} \sigma_{1} / \gamma_{1} \sigma_{2}, \zeta_{1}\right\}$, the value function of the game is a decreasing $C^{2}$ function given by

$$
V(x)= \begin{cases}\frac{G_{1}\left(x_{1}, x\right)}{C_{4}}, & x_{1}<x<\infty, \\ 1-\frac{H_{2}\left(x_{1}, x\right)}{C_{4}}, & -\infty<x \leq x_{1},\end{cases}
$$

where $C_{4}=H_{2}\left(x_{1}, x_{1}\right)+G_{1}\left(x_{1}, x_{1}\right)$, and the Nash equilibrium strategy is the feedback control associated with the risk exposure functions

$$
\left(a^{*}(x), b^{*}(x)\right)= \begin{cases}\left(-\frac{\gamma_{1}}{\sigma_{1}^{2}} f_{1}(x)+\rho \frac{\sigma_{2}}{\sigma_{1}}, 1\right), & x_{1}<x<\infty, \\ (1,1), & -\infty<x \leq x_{1} .\end{cases}
$$

Remark 4.5. The results in (4.13) and Theorem 4.3 also hold if $\max \left\{\sigma_{2} / \sigma_{1}, \gamma_{2} \sigma_{1} / \gamma_{1} \sigma_{2}\right\}<\rho<$ $\min \left\{\frac{1}{2} \sigma_{1} / \sigma_{2}, \zeta_{1}\right\}$. We note that the parameter conditions under which the results in Theorem 4.3 hold can be found by Lemmas 4.1, 4.2, and 4.6.

We have discussed several parameter cases under which the game is solvable with the value function and Nash equilibrium reinsurance strategy given explicitly. For other parameter cases with $\rho>0$, we observe that the sets $\mathcal{R}_{1}, \ldots, \mathcal{R}_{8}$ have endpoints that are not commonly shared. The sets $\mathcal{R}_{0}, \mathcal{R}_{1}, \ldots, \mathcal{R}_{8}$ do not connect to form a partition of the real line. Thus, in these cases, the game is not solvable and the value function may not exist.

\section{Conclusion}

From the explicit results, we observed that the value function $V$ is z-shaped and has a unique reflection point at $x_{2}$ (concave below $x_{2}$ and convex above) in all of the solvable cases. The risk exposure functions of the Nash equilibrium strategy are continuous and monotone. From the optimal play, we showed that insurer one never buys reinsurance when the surplus difference is below $x_{2}$ and insurer two never buys reinsurance when the difference is above $x_{2}$. Also, as the surplus difference increases, the optimal risk exposure decreases for insurer one and increases for insurer two. This implies that, when any insurer finds themselves in a better position, the 
insurer takes less risk and buys more reinsurance; if an insurer finds themselves in a more disadvantaged position, the insurer holds a bolder strategy by taking more risk and buying less reinsurance. This feature of the optimal reinsurance control has been seen in ruin and absolute ruin minimization problems (see, e.g. [4] and [7]) and also in the exit probability game of [9]. The relationship between an insurer's position and their willingness of risk-taking is consistent with that in the investment game of [1].

We showed that the reinsurance game is solvable when certain parameter conditions are satisfied (negative correlation, small positive correlation, and some other special cases). The value function and the Nash equilibrium reinsurance strategy were found by solving the FBI equations explicitly in these cases. We note that, for the other parameter cases, the game is not solvable and the value function may not exist. Future studies on sup-value and sub-value functions might be suited to proving the nonexistence of the value function when the game is not solvable. However, in this research we focused on only the solvable cases. We also note that in this paper absolute dominance was introduced for the first time as a win scenario in insurance game theory. The performance function was defined as the probability of absolute dominance and only proportional reinsurance control was considered. In future research, one may apply other scenarios to define the performance function and include investment, dividend, or nonproportional reinsurance in the game.

\section{References}

[1] Browne, S. (2000). Stochastic differential portfolio games. J. Appl. Prob. 37, 126-147.

[2] Emanuel, D. C., Harrison, J. M. and Taylor, A. J. (1975). A diffusion approximation for the ruin function of a risk process with compounding assets. Scand. Actuarial J. 1975, 240-247.

[3] Fleming, W. H. and Souganidis, P. E. (1989). On the existence of value functions of two-player, zero-sum stochastic differential games. Indiana Univ. Math. J. 38, 293-314.

[4] LuO, S. AND TAKSAR, M. (2011). On absolute ruin minimization under a diffusion approximation model. Insurance Math. Econom. 48, 123-133.

[5] Mataramvura, S. and ØKSendal, B. (2008). Risk minimizing portfolios and HJBI equations for stochastic differential games. Stochastics 80, 317-337.

[6] Schmidui, H. (1994). Diffusion approximations for a risk process with the possibility of borrowing and investment. Commun. Statist. Stoch. Models 10, 365-388.

[7] TAKsar, M. and Markussen, C. (2003). Optimal dynamic reinsurance policies for large insurance portfolios. Finance Stoch. 7, 97-121

[8] TAKSAR, M. AND ZENG, X. (2011). Optimal non-proportional reinsurance control and stochastic differential games. Insurance Math. Econom. 48, 64-71.

[9] Zeng, X. (2010). A stochastic differential reinsurance game. J. Appl. Prob. 47, 335-349. 